\title{
Quantized phenomena in graphene nanoripples
}

\author{
Feng Liu*, Tzu-Chiang Wang \\ State Key Laboratory of Nonlinear Mechanics, Institute of Mechanics, Chinese Academy of Sciences, Beijing 100190, People's Republic of China
}

\section{A R T I C L E I N F O}

\section{Article history:}

Received 9 June 2015

Received in revised form

16 October 2015

Accepted 21 October 2015

Available online 23 October 2015

\begin{abstract}
A B S T R A C T
In this study, a previously published continuum model for a stretched sheet was adapted to the case of a compressed sheet to understand the geometry of graphene nanoripples. The validity of the model is tested by comparing with experimental data. Furthermore, the existence of an intrinsic wavelength is revealed based on a simple mechanical analysis, and is confirmed by the quantized feature of the experimental data. Although only graphene nanoripples are investigated in this study, it is believed that such quantized phenomena could exist in other two-dimensional (2D) materials and even in superthin films. Thus, it is believed that the model proposed in this study can help develop the control of nanoripples as an engineering method for future applications.
\end{abstract}

(C) 2015 Elsevier Ltd. All rights reserved.

\section{Introduction}

Although graphene has attracted enormous theoretical interest because of its linear energy-momentum dispersion relations crossing at the Dirac point [1,2], to open an electrical band gap is a fundamental obstacle for its applications in semiconductor industry. Several studies are devoted to meet this goal using the quantum confinement effect [3], chemical modification [4,5], periodical structural modification [6-10], and substrate modification [11,12]. However, all these methods lack controllability.

Recently, a pseudo-magnetic field concept has been introduced $[13,14]$, which demonstrates that a nonuniform strain field could be effectively viewed as a magnetic field, and thus provide a new route to open a band gap in graphene. Controllability could be expected, as the strain can be controlled by external loads. Without much effort, the corresponding pseudo-Landau levels in graphene were experimentally observed [15-19], particularly the magnitude of the nearly uniform pseudo-magnetic field was found to be $>300 \mathrm{~T}$ for a nanobubble [15]. However, the controlled formation of a certain nanostructure in graphene is almost impossible, and therefore could not be used as a conventional method to open an electrical band gap in graphene. Besides, F. Guinea et al. suggested that designed loads could generate a uniform pseudo-magnetic field [20], but the technical difficulties made the experiment complex. When graphene is attached to a substrate, ripples could be generated via thermal processing, because of the compressing instability

\footnotetext{
* Corresponding author.

E-mail address: liufeng@imech.ac.cn (F. Liu).
}

of thin films [21-24] (considering the negative thermal expansion coefficient of graphene compared with the positive one of the substrate). Because of the nonuniform strain field induced by ripples, and considering the periodicity of ripples, there will be a large electrical band gap [24]. Particularly, the size of ripples and thus the corresponding band gap could be controlled by the macro-thermal processing, making the method quite attractive. Accordingly, the geometry of ripples under external loads becomes an important issue to be considered, which has been widely investigated [25-30]. Until now, the model established by E. Cerda and L. Mahadevan has been used to explain the wavelength and amplitude of ripples [31]. Although most experimental data agree fairly well with this model, recently, large discrepancies have been reported for graphene nanoripples [21,24]. Because nanoripples would lead to a larger nonuniform strain, a larger electrical band gap would result, which is of great importance to understand this behavior.

From a theoretical viewpoint, as the model for a stretched sheet has been established [31], the one for a compressed sheet is still missing. In this article, a continuum model for a compressed sheet was proposed to help understand the geometry of graphene nanoripples. The comparisons of the experimental data, existing models, and the proposed model demonstrate the validity of the model. Furthermore, on the basis of a simple mechanical analysis, the intrinsic wavelength of graphene ripples was provided. The existence of the intrinsic wavelength was confirmed by comparing with the experimental data. This further confirms that the model proposed in this study can help develop the control of nanoripples as an engineering method for future applications. 


\section{Model}

Let us consider a compressed sheet with two ends fixed (Fig. 1(b); the schematic of the model developed by E. Cerda and L. Mahadevan [31] is shown in Fig. 1(a) for comparison). The out-ofplane displacement of the sheet, that is, deflection, is $\xi(x, y)$, where $0<x<L$ and $0<y<W$. Comparing with the ripples generated in a stretched sheet, ripples in our model are induced by compression along the $y$ direction. Because of the discrepancy of physical origins, the physical meaning of the stretching energy is quite different: the stretching energy is ascribed to the work done by the axial tension [31], whereas in our model, the stretching energy is derived from the elastic energy because of the $x$ directional tensile force (considering the two fixed ends) induced by the deflection as a result of the compression along $y$ direction. However, the wavelength and amplitude of the ripples are yet to be determined by considering the bending and stretching energies and an incompressibility condition in the energy functional as follows [31]:

$U_{t o t}=U_{B}+U_{S}-L$

where $U_{B}=(1 / 2) \int_{A} B\left(\partial_{y}^{2} \xi\right)^{2} d A$ is the bending energy due to the deflection in the $y$ direction, where $B=E t^{3} /\left[12\left(1-\nu^{2}\right)\right]$ is the bending stiffness, and $U_{S}=E t \varepsilon_{X} A$ is the stretching energy, where $\varepsilon_{X}=\int_{A}(1 / 2)\left(\partial_{x} \xi\right)^{2} d A / A$ is the average strain along the $x$ direction. It is important to note that this stretching energy is quite different from the one defined by E. Cerda and L. Mahadevan [31]. Let us assume that the sheet satisfies the condition of incompressibility along the $y$ direction, that is

$\int_{0}^{W}\left[\frac{1}{2}\left(\partial_{y} \xi\right)^{2}-\frac{\Delta}{W}\right] d y=0$

which leads to the last term in Eq. (1), $L=\int_{A} \beta\left[\left(\partial_{y} \xi\right)^{2} / 1-\Delta / W\right] d A$, and implements this constraint through a Lagrange multiplier $\beta$, with $\Delta=\varepsilon_{y} W$ ( $\varepsilon_{y}$ is the strain along the $y$ direction) as the compressive displacement along the $y$ direction. The Euler-Lagrange equation corresponding to the energy functional Eq. (1) (i.e., $\delta U_{\text {tot }} / \delta \xi=0$ ) can be obtained as

$B \partial_{y}^{4} \xi-T \partial_{x}^{2} \xi+\beta \partial_{y}^{2} \xi=0$

Although the form of the Euler-Lagrange equation is the same, the definition of $T$ is different, which is $T=E t$ as opposed to $T=E t \varepsilon_{x}$ [31]. A periodic solution of the form $\xi=e^{i k_{n} y} X_{n}(x)$ is assumed and substituted into Eq. (3) as $\frac{d^{2} X_{n}}{d x^{2}}+\omega_{n}^{2} X_{n}=0, \quad X_{n}(0)=X_{n}(L)=0$,

where $\omega_{n}^{2}=\left(\beta k_{n}^{2}-B k_{n}^{4}\right) / T$. The solution to the above equation is $X_{n}=A_{n} \sin \omega_{n} \chi, \omega_{n}=m \pi / L$ (where $m$ is a positive integer), and when $m=1$, the bending energy is minimum, and hence $\beta=\left(\pi^{2} T / L^{2} k_{n}^{2}\right)+B k_{n}^{2}$ and $\xi=A_{n} \cos \left(k_{n} y+\varphi_{n}\right) \sin \pi x / L$. Substituting this solution into Eq. (2) with $W \cdot k_{n} \gg 1$ (as in experiments) and averaging over $x$ yields $A_{n}^{2} k_{n}^{2} W / 8 \approx \Delta$ (the details of this derivation is provided in Supplementary Information), establishing a correlation between wave number and amplitude, with which the total energy could be simplified as $U_{\text {tot }}=B k_{n}^{2} \Delta L+\pi^{2} T \Delta / k_{n}^{2} L$. By minimizing $U_{\text {tot }}$ with respect to $k_{n}$, the wavelength, $\lambda=2 \pi / k_{n}$, and amplitude, $A_{n}$, could be determined as follows:

$\lambda=2 \sqrt{\pi}\left(\frac{B}{T}\right)^{1 / 4} L^{1 / 2}$ and $A_{n}=\frac{\sqrt{2}}{\pi}\left(\frac{\Delta}{W}\right)^{1 / 2} \lambda$.

By substituting the values of $B$ and $T$, the wavelength and amplitude can be calculated as

$\lambda=\frac{(2 \pi L t)^{1 / 2}}{\left[3\left(1-\nu^{2}\right)\right]^{1 / 4}} \quad$ and $\quad A_{n}=\left(\varepsilon_{y} L t\right)^{1 / 2}\left[\frac{16}{3 \pi^{2}\left(1-\nu^{2}\right)}\right]^{1 / 4}$.

It is important to note that in our model, $T=E t$ is much larger than $T=E t \varepsilon_{x}$ [31], when $\varepsilon_{x} \ll 1$. Hence, according to Eq. (5), both the wavelength $\lambda$ and amplitude $A_{n}$ are inversely proportional to $T^{1 / 4}$. The previous models overestimate the wavelength and the amplitude for nanoripples because of the smaller value of $T$ (weak resistance to stretch), which becomes much larger in the proposed model. Therefore, a better performance could be expected from our model.

\section{The graphene sheet attached to a substrate}

The system we would like to study is the graphene sheet attached to a substrate, which is slightly different from the suspended sheet analyzed above. The expression of $\xi$ is now written as $\xi=A_{n}\left[\cos \left(k_{n} y+\varphi_{n}\right)+1\right] \sin \pi x / L$, such that the valleys are always attached to the substrate, and the corresponding geometry can be found in Fig. 2. It is important to note that it is not the solution of Eq. (3), but by imposing the condition of incompressibility and minimizing the total energy, the wavelength and amplitude could again be obtained as:

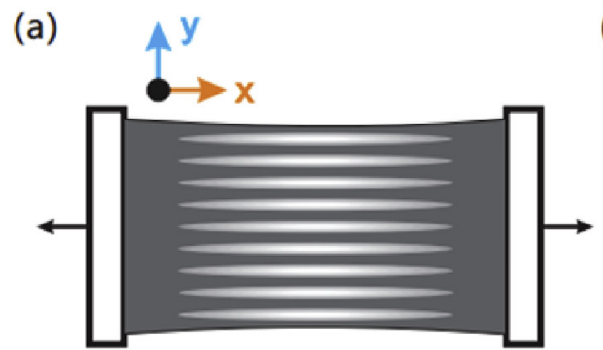

(b)

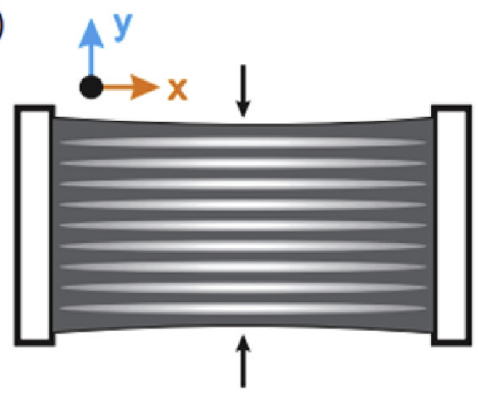

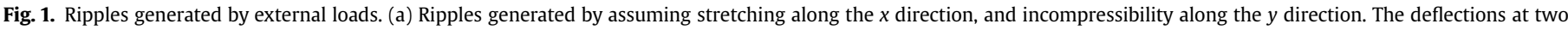

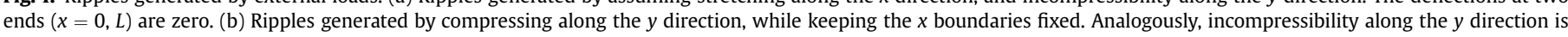

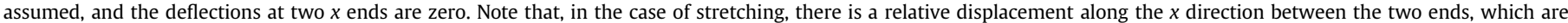

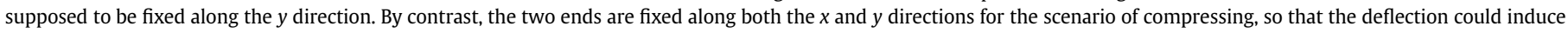
tensile strain in the $x$ direction. (A color version of this figure can be viewed online.) 
(a)

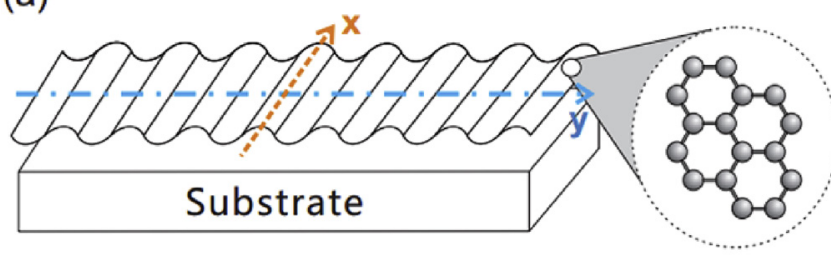

(b)

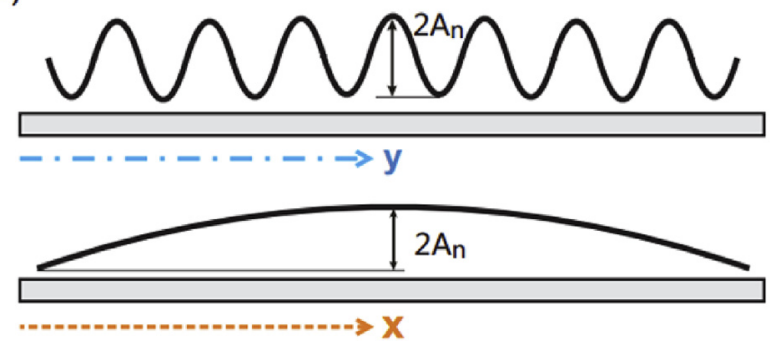

Fig. 2. (a) Geometry of graphene ripples on a substrate. Part of ripples is zoomed out, and $x$ and $y$ directions are marked, consistent with the previous definition in Fig. 1. (b) The sections at the position of directions $x$ and $y$ marked in (a) are shown to help understand the expression of the defection given in the context. (A color version of this figure can be viewed online.)

$\lambda=\frac{(2 \pi L t)^{1 / 2}}{\left[9\left(1-\nu^{2}\right)\right]^{1 / 4}} \quad$ and $\quad A_{n}=\left(\varepsilon_{y} L t\right)^{1 / 2}\left[\frac{16}{9 \pi^{2}\left(1-\nu^{2}\right)}\right]^{1 / 4}$

In the experiments, several groups have carefully investigated and confirmed the classical membrane nature of graphene [22,23]; in other words, the geometry of graphene ripples is consistent with the model proposed by E. Cerda and L. Mahadevan [31]. However, recently, large discrepancies between the characteristics of smaller nanoripples and the model proposed in Ref. [31] have been reported $[21,24]$. Further, it has been found that the wavelength or amplitude predicted by this model could be one order larger than the experimental observation, and hence it is thought to be a breakdown of the continuum mechanics for graphene nanoripples. With constant $L$, the wavelength and amplitude of graphene

Table 1

Model comparison. With constant $L$, the results of the original model of E. Cerda and L. Mahadevan [31] (model 1), the modified one for compressing [26] (model 2), and our model are provided and compared with two experimental data of graphene nanoripples [21,24] (exp 1 and exp 2). It is important to note that $t=0.08 \mathrm{~nm}[21,24]$ and $\nu=0.16[21,24]$ are used in all of these calculations. For model $1, \varepsilon_{X}=0.02$ (the case of stretching, Fig. 1(a)); for model 2, $\varepsilon_{y}=0.02$ indicating $\varepsilon_{x}=\varepsilon_{y} \times \nu=0.0032$ (equivalent to the case of stretching); and for our model $\varepsilon_{y}=0.02$ (the case of compressing, Fig. 1(b)). Although the Poisson's ratio $\nu$ is predicted to range from 0.1 to 0.3 for single-layer graphene [32-34], different choices have little effect on the results. Besides, the strain produced by thermal processing is estimated at about 0.02 both for experiments 1 and 2 through the similar arguments in Ref. [21].

\begin{tabular}{llll}
\hline & $\lambda(\mathrm{nm})$ & $A_{n}(\mathrm{~nm})$ & $L(\mathrm{~nm})$ \\
\hline Exp 1 & 0.7 & 0.05 & 5.0 \\
model 1 & 3.2 & 0.08 & - \\
model 2 & 2.0 & 0.13 & - \\
Our results & 0.92 & 0.059 & - \\
& & & $400-1000$ \\
Exp 2 & $3.0-8.0$ & $0.2-0.7$ & - \\
model 1 & $28.8-45.6$ & $0.73-2.21$ & - \\
model 2 & $18.2-28.8$ & $1.16-1.83$ & - \\
Our results & $8.2-13.0$ & $0.52-0.83$ & \\
\hline
\end{tabular}

nanoripples calculated by the existing and proposed models are provided and compared with the experimental data in Table 1 [21,24]. It is evident from the table that the proposed model agrees much better with experimental measurements. However, there is still a discrepancy between our predictions and the experimental measurements [24], which could be attributed to the presence of nitrogen dopants in graphene.

A question that needs to be answered is "why experiments seem to obey different rules?" The answer, we believe, is irrelevant to the size of ripples, but determined by the boundary condition along the axial direction of ripples. According to Refs. [22,23], the two ends along the axial direction of ripples are not fixed, and thus our model is not suitable for that case. However, it could be well described by the modified model of E. Cerda and L. Mahadevan, which has been proved numerically by MD simulation [26]. However, nanoripples $[21,24]$ produced by thermal processing are localized in such a way that they are surrounded by unwrinkling graphene or other nanoripples, which impede deformation along the axial direction of ripples, and thus could be considered as the fixed boundaries (i.e., the two fixed ends) in our model.

\section{Intrinsic wavelength}

In fact, of all these experimental measurements, $0.7 \mathrm{~nm}$ is the minimum wavelength of ripples has ever been found in graphene [21]. A problem has arisen: Is there a minimum or intrinsic wavelength? In order to find the minimum or intrinsic wavelength in ripples, the system must be studied at the beginning of compression, that is, at $\varepsilon_{y} \sim 0$. This is because, if the adsorption energy between the graphene sheet and the substrate is considered, the wavelength should increase gradually as the compressing strain becomes larger. Then, if there is an intrinsic wavelength, it is determined by the balance between the bending energy and adsorption energy. Thus, the total energy is calculated as

$U_{\text {tot }}=U_{B}+U_{A}$,

where $U_{B}=(1 / 2) \int_{A} B\left(\partial_{y}^{2} \xi\right)^{2} d A$ and $U_{A}$ represent the bending energy and the adsorption energy, respectively. Although the bending energy is easy to calculate, the calculation of adsorption energy is relatively complex, as it has no analytical expression. Thus, it is calculated self-consistently using the projector augmented wave pseudo-potential method implemented in the VASP package [35]. The values of k-mesh, energy cutoff, and convergence criteria are set to be $27 \times 27 \times 1,420 \mathrm{eV}$, and $10^{-5} \mathrm{eV}$, respectively, and the maximum allowed force on each atom for structure optimization is $<10^{-3} \mathrm{eV} / \AA$. Considering the van der Waals interaction between graphene and the substrate, various exchange-correlation potentials are used and compared to confirm our results.

The adsorption energy is expressed as

$E_{a d}=E_{\text {total }}-E_{G r}-E_{C u}$,

where $E_{\text {total }}, E_{G r}$, and $E_{C u}$ represent the energies of the whole system, monolayer graphene, and substrate, respectively. The calculation model is same as that in Ref. [36], where one primitive cell is used for graphene with three layers of $\mathrm{Cu}(111)$ substrate below (Fig. 3(a) and (b)), and the in-plane lattice constant will be forced to the LDAoptimized value of graphene, that is, $a=2.445 \AA$. The adsorption energies of graphene on the $\mathrm{Cu}(111)$ surface calculated with LDA [37], PBE [38], DFT-D2 [39] (PBE is chosen to be the exchangecorrelation functional), and vdW-DF [40-42] functionals with respect to the interlayer distance are shown in Fig. 3(c). Compared with LDA and PBE, the long-range van der Waals interaction has been considered in DFT-D2 and vdW-DF, and adsorption energy 
(a)

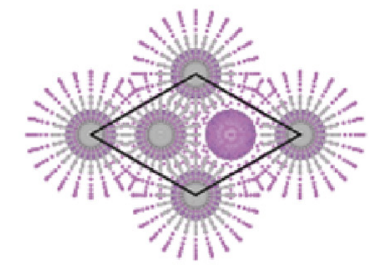

(b)

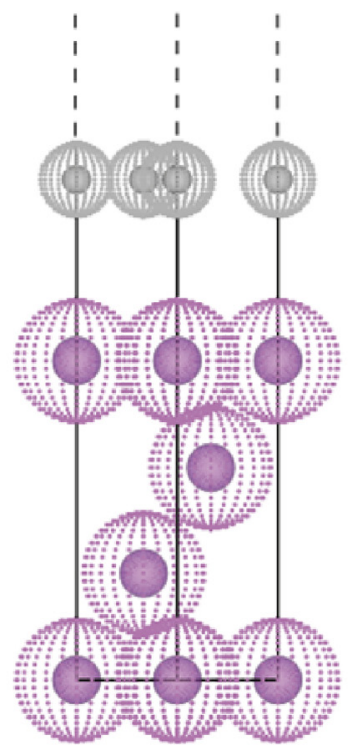

(c)

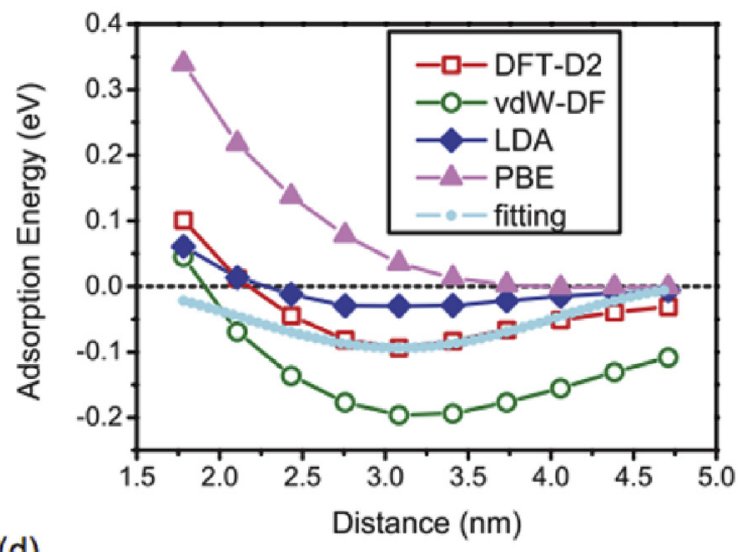

(d)

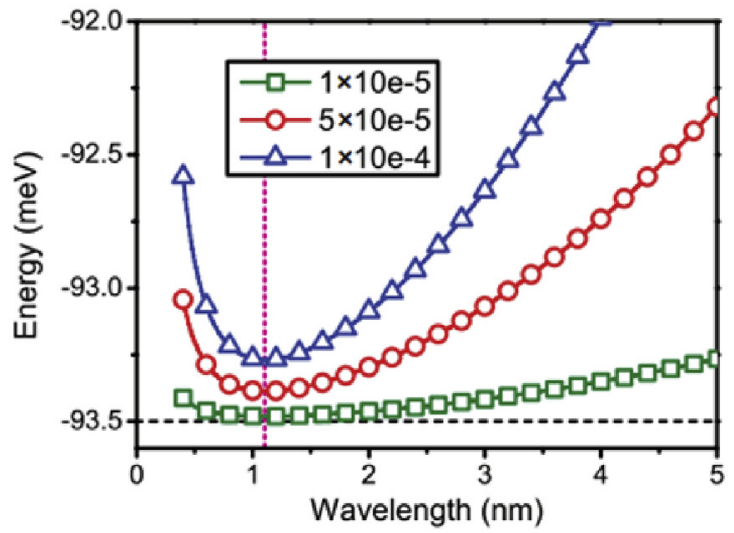

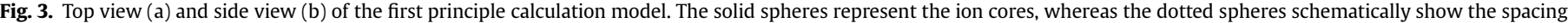

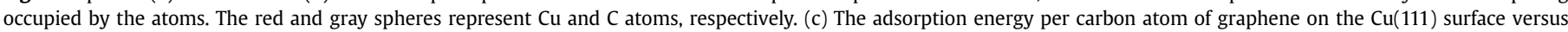
interlayer distance. (d) Total energy versus wavelength for $\varepsilon_{y}=1 \times 10^{-5}, 5 \times 10^{-5}$, and $1 \times 10^{-4}$. (A color version of this figure can be viewed online.)

almost larger than one order is obtained. A simple analytical expression

$E_{a d}(r)=-\frac{E_{\min }}{2} \cdot \sin \left(\frac{\pi\left(r-d_{e}\right)}{\left(d_{c}-d_{e}\right)}-\frac{\pi}{2}\right)+\frac{E_{\min }}{2}$

is used to fit the result of DFT-D2 (which has been systematically tested, and a better performance than vdW-DF for layered structures is resulted [43]), where the minimum adsorption energy $E_{\min }=-0.0935 \mathrm{eV} /$ atom (equivalent to $-0.0357 \mathrm{eV} / \AA^{2}$ ), the equilibrium distance $d_{e}=3.0829 \AA$, and the cutoff distance $d_{c}=5.0 \AA$. It can be observed in Fig. 3(c) that the analytical expression provided above fits the adsorption energy fairly well near the equilibrium distance, and is sufficient for investigating the scenario of $\varepsilon_{y} \sim 0$.

Assuming $\xi=A_{n}[\sin (2 \pi y / \lambda)+1]+d_{e}$ and incompressibility along the $y$ direction (Eq. (2)), the total energy (Eq. (9)) could be directly calculated with the bending stiffness $B=1.4 \mathrm{eV}$ [44]. Three values of strain along the $y$ direction $\varepsilon_{y}\left(10^{-5}, 5 \times 10^{-5}\right.$, and $\left.10^{-4}\right)$ are used to calculate the total energy $U_{\text {tot, }}$ and it is obvious that although the minimum of the total energy is changed, the corresponding wavelength about $1.1 \mathrm{~nm}$ remains unchanged (Fig. 3(d)). In fact, with $\varepsilon_{y} \sim 0$ and assuming the a finite wavelength, $\lambda$, the intrinsic wavelength could be analytically obtained as

$\lambda=\left[\frac{32 \pi^{2} B\left(d_{c}-d_{e}\right)^{2}}{-3 E_{\min }}\right]^{1 / 4}$.
With the values of the parameters substituted, this equation gives $\lambda=1.11 \mathrm{~nm}$, which agrees with our numerical result. In fact, it is easy to prove that the existence of the intrinsic wavelength is universal, if the harmonic approximation is accurate for the adsorption energy (Eq. (9)) near its minimum.

Analogous to the balance of the potential energy between atoms determining the interatomic distance, the competition between the bending energy and adsorption energy here would provide a length scale (i.e., the intrinsic wavelength) during thermal processing. As a consequence, the wavelength of ripples could be quantized. In Fig. 4(c), the experimental measurements of the wavelength of ripples are reorganized, and the quantized feature is apparent, that is, most of the wavelengths are integer times of $0.7 \mathrm{~nm}$ (the minimum wavelength has been observed in the experiment as mentioned earlier), which is smaller than the predicted value, $1.1 \mathrm{~nm}$. However, if we use the binding energy obtained by the $\mathrm{vdW}-\mathrm{DF}$, the predicted intrinsic wavelength is $0.93 \mathrm{~nm}$, much closer to the experimental value $0.7 \mathrm{~nm}$, which indicates that a lack of accurate estimation of the van der Waals interaction in first principle calculation may contribute to this difference. In addition to the integer times of $0.7 \mathrm{~nm}$, there are a few wavelengths being half-integer times of $0.7 \mathrm{~nm}$ lacking a rational answer (Fig. 4(c)). In fact, if the wavelength of the new generated ripples is assumed to cover the equivalent underlying structure (i.e., ripples with the intrinsic wavelength plotted with black solid lines in Fig. 4(a) and (b)), there are two alternative schemes. The first one is to cover the same underlying structure, that is, the wavelength of the new 
(a)

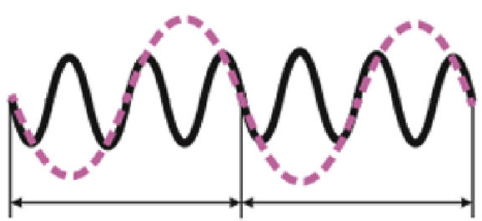

(b)

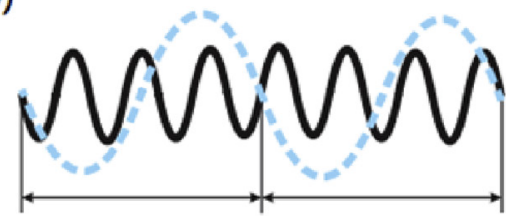

(c)

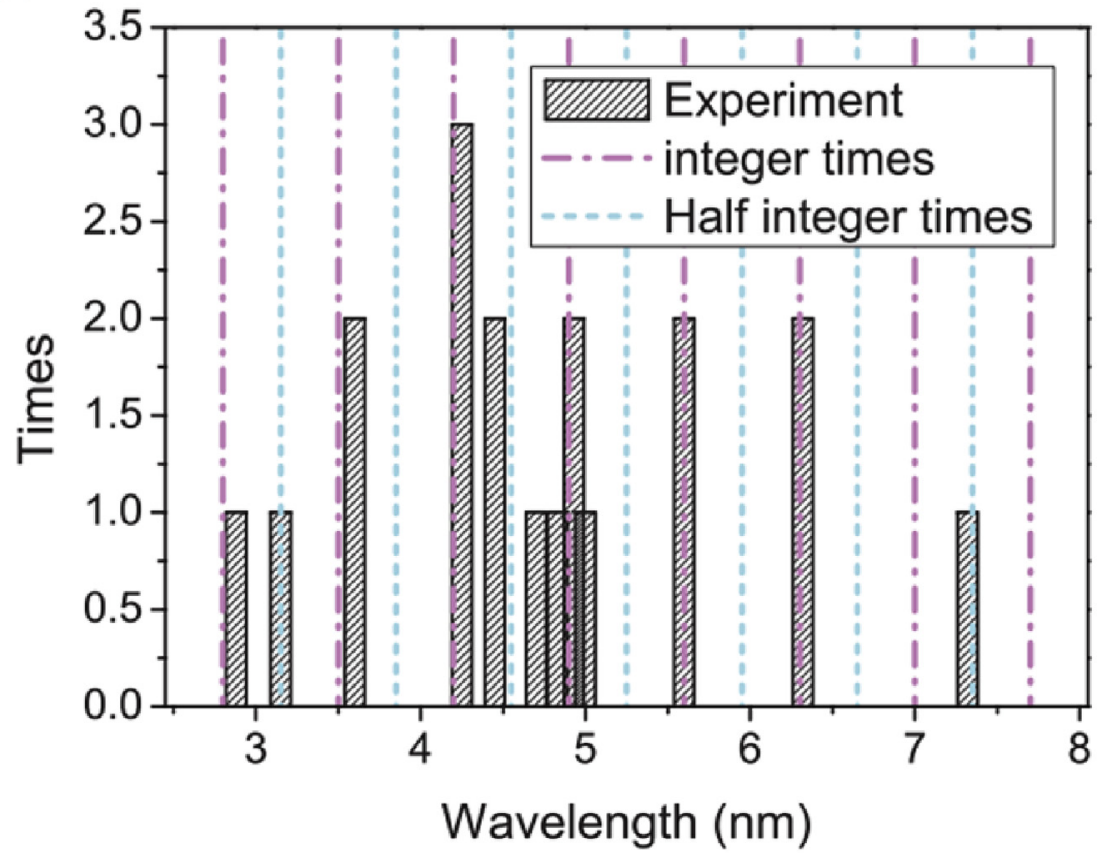

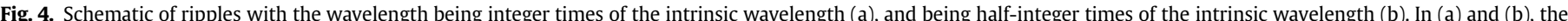

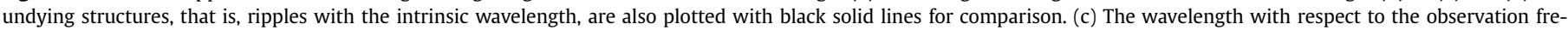

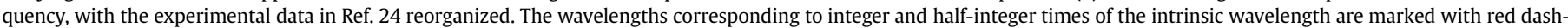

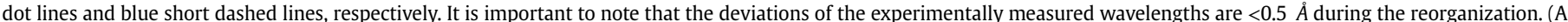
color version of this figure can be viewed online.)

generated ripples equals the integer times of the intrinsic wavelength (Fig. 4(a)). The second one is to make the wavelength of the new generated ripples equal to half-integer times of the intrinsic wavelength (Fig. 4(b)). Although the underlying structures are different, they are linked by the spatial inversion, and thus are thought to be equivalent. The observation frequency of the experiment is shown in Fig. 4(c), which indicates that the first scheme is preferred over the second one during thermal processing. Besides, it is speculated that the deviations of the integer and half-integer times of the intrinsic wavelength possibly stem from nitrogen dopants in graphene, as mentioned earlier.

In order to understand this quantized phenomenon, it should be noted that the intrinsic or minimum wavelength is present at the beginning of the deformation $\left(\varepsilon_{y} \sim 0\right)$. Then, with the increasing deformation, the wavelength will become large. However, because of the existence of the intrinsic wavelength, the characteristic length of the structural rearrangement could be estimated by considering the compatibility of two wavelengths, that is

$m \cdot \lambda_{\text {nano }}=n \cdot \lambda_{\text {in }} / 2$,

where $m$ and $n$ are integers, $\lambda_{\text {nano }}$ is the wavelength of the nanoripple, and $\lambda_{\text {in }}$ is the intrinsic wavelength. If the wavelength of the nanoripple is integer or half-integer multiple of the intrinsic wavelength, the system could rearrange locally to generate the nanoripple, because in this case $m$ could be equal to 1 . However, if the wavelength obtained by the energy minimization slightly deviates $(\Delta \lambda \rightarrow 0)$ from the integer or half-integer multiple of the intrinsic wavelength,

$m \cdot \lambda_{\text {nano }}=n \cdot \lambda_{\text {in }} / 2, \quad \lambda_{\text {nano }}=n_{1} \cdot \lambda_{\text {in }} / 2+\Delta \lambda$,

where $n_{1}$ is an integer and the minimum value of $m$ is $\lambda_{\text {in }} / 2 \Delta \lambda$, so that the system rearranges globally to generate this nanoripple. In fact, this is not possible, because there is a lack of long-range and relatively strong interaction within the system to provide the spontaneous global structural adjustment. Therefore, the system deforms rather heterogeneously, so that only local rearrangements are required to achieve a local optimized configuration, where the local wavelengths are always integer or half-integer multiple of the intrinsic wavelength. Indeed, in the experiment for nanoripples, different wavelengths could be observed in one sample (Fig. 2 in Ref. [24]), indicating that the system deforms heterogeneously, and almost all the wavelengths of these nanoripples (Fig. 4) are integer or half-integer multiple of the intrinsic wavelength.

\section{Conclusion}

In this article, we first proposed a continuum model for a compressed sheet, which well described the geometry of graphene nanoripples. The effect of differing boundary conditions is emphasized, and is believed to be the reason that experimental 
measurements of ripples seem to obey different rules. Furthermore, the existence of an intrinsic wavelength is revealed based on a simple mechanical analysis, and is confirmed by the quantized behavior of the experimental data. Although only graphene nanoripples are investigated in this study, quantized phenomena could exist in other two-dimensional (2D) materials and even in superthin films. Thus, it is believed that the model proposed in this study can help develop the control of nanoripples as an engineering method for future applications.

\section{Acknowledgments}

This study was supported by the National Natural Science Foundation of China (Grant Nos. 11021262 and 11172303) and the National Basic Research Program of China ("973” Project) (Grant No. 2012CB937500).

\section{Appendix A. Supplementary data}

Supplementary data related to this article can be found at http:// dx.doi.org/10.1016/j.carbon.2015.10.070.

\section{References}

[1] A.K. Geim, K.S. Novoselov, The rise of graphene, Nat. Mater. 6 (3) (2007) 183-191.

[2] A.H. Castro Neto, F. Guinea, N.M.R. Peres, K.S. Novoselov, A.K. Geim, The electronic properties of graphene, Rev. Mod. Phys. 81 (1) (2009) 109-162.

[3] Y.W. Son, M.L. Cohen, S.G. Louie, Energy gaps in graphene nanoribbons, Phys. Rev. Lett. 97 (21) (2006) 216803.

[4] R. Balog, B. J?rgensen, L. Nilsson, M. Andersen, E. Rienks, M. Bianchi, et al., Bandgap opening in graphene induced by patterned hydrogen adsorption, Nat. Mater. 9 (4) (2010) 315-319.

[5] L. Ci, L. Song, C. Jin, D. Jariwala, D. Wu, Y. Li, et al., Atomic layers of hybridized boron nitride and graphene domains, Nat. Mater. 9 (5) (2010) 430-435.

[6] M.T. Lusk, L.D. Carr, Nanoengineering defect structures on graphene, Phys. Rev. Lett. 100 (17) (2008) 175503.

[7] J. Lahiri, Y. Lin, P. Bozkurt, Oleynik II, M. Batzill, An extended defect in graphene as a metallic wire, Nat. Nanotechnol. 5 (5) (2010) 326-329.

[8] J. Eroms, D. Weiss, Weak localization and transport gap in graphene antidot lattices, New J. Phys. 11 (9) (2009) 095021.

[9] J. Bai, X. Zhong, S. Jiang, Y. Huang, X. Duan, Graphene nanomesh, Nat. Nanotechnol. 5 (3) (2010) 190-194.

[10] M. Kim, N.S. Safron, E. Han, M.S. Arnold, P. Gopalan, Fabrication and characterization of large-area, semiconducting nanoperforated graphene materials, Nano Lett. 10 (4) (2010) 1125-1131.

[11] S. Zhou, G.H. Gweon, A. Fedorov, P. First, W. De Heer, D.H. Lee, et al., Substrateinduced bandgap opening in epitaxial graphene, Nat. Mater. 6 (10) (2007) $770-775$.

[12] J. Hicks, A. Tejeda, A. Taleb-Ibrahimi, M. Nevius, F. Wang, K. Shepperd, et al., A wide-bandgap metal-semiconductor-metal nanostructure made entirely from graphene, Nat. Phys. 9 (1) (2013) 49-54.

[13] M. Vozmediano, M. Katsnelson, F. Guinea, Gauge fields in graphene, Phys. Rep. 496 (4) (2010) 109-148.

[14] F. Guinea, M. Katsnelson, A. Geim, Energy gaps and a zero-field quantum hall effect in graphene by strain engineering, Nat. Phys. 6 (1) (2010a) 30-33.

[15] N. Levy, S.A. Burke, K.L. Meaker, M. Panlasigui, A. Zettl, F. Guinea, Straininduced pseudo-magnetic fields greater than 300 tesla in graphene nanobubbles, Science 329 (5991) (2010) 544-547.

[16] H. Yan, Y. Sun, L. He, J.C. Nie, M.H.W. Chan, Observation of landau-level-like quantization at $77 \mathrm{k}$ along a strained-induced graphene ridge, Phys. Rev. B 85 (3) (2012) 035422.

[17] D. Guo, T. Kondo, T. Machida, K. Iwatake, S. Okada, J. Nakamura, Observation of landau levels in potassium-intercalated graphite under a zero magnetic field, Nat. Commun. 3 (2012) 1068.
[18] L. Meng, W.Y. He, H. Zheng, M. Liu, H. Yan, W. Yan, et al., Strain-induced onedimensional landau level quantization in corrugated graphene, Phys. Rev. B 87 (20) (2013a) 205405.

[19] W. Yan, W.Y. He, Z.D. Chu, M. Liu, L. Meng, R.F. Dou, et al., Strain and curvature induced evolution of electronic band structures in twisted graphene bilayer, Nat. Commun. 4 (2013) 2159.

[20] F. Guinea, M.I. Katsnelson, A.K. Geim, Energy gaps and a zero-field quantum hall effect in graphene by strain engineering, Nat. Phys. 6 (1) (2010b) 30-33.

[21] L. Tapasztó, T. Dumitrică, S.J. Kim, P. Nemes-Incze, C. Hwang, L.P. Biró, Breakdown of continuum mechanics for nanometre-wavelength rippling of graphene, Nat. Phys. 8 (10) (2012) 739-742.

[22] W. Bao, F. Miao, Z. Chen, H. Zhang, W. Jang, C. Dames, et al., Controlled ripple texturing of suspended graphene and ultrathin graphite membranes, Nat. Nanotechnol. 4 (9) (2009) 562-566.

[23] L. Meng, Y. Su, D. Geng, G. Yu, Y. Liu, R.F. Dou, et al., Hierarchy of graphene wrinkles induced by thermal strain engineering, Appl. Phys. Lett. 103 (25) (2013b) 251610.

[24] K.K. Bai, Y. Zhou, H. Zheng, L. Meng, H. Peng, Z. Liu, et al., Creating onedimensional nanoscale periodic ripples in a continuous mosaic graphene monolayer, Phys. Rev. Lett. 113 (8) (2014) 086102.

[25] C. Wang, Y. Liu, L. Lan, H. Tan, Graphene wrinkling: formation, evolution and collapse, Nanoscale 5 (10) (2013b) 4454-4461.

[26] Z. Wang, M. Devel, Periodic ripples in suspended graphene, Phys. Rev. B 83 (12) (2011) 125422

[27] T.H. Fang, W.J. Chang, K.P. Lin, S.T. Shen, Stability and wrinkling of defective graphene sheets under shear deformation, Curr. Appl. Phys. 14 (4) (2014) 533-537.

[28] C. Wang, L. Lan, H. Tan, The physics of wrinkling in graphene membranes under local tension, Phys. Chem. Chem. Phys. 15 (8) (2013a) 2764-2773.

[29] Z. Zhang, W.H. Duan, C.M. Wang, Tunable wrinkling pattern in annular graphene under circular shearing at inner edge, Nanoscale 4 (16) (2012) 5077-5081.

[30] W.H. Duan, K. Gong, Q. Wang, Controlling the formation of wrinkles in a single layer graphene sheet subjected to in-plane shear, Carbon 49 (9) (2011) 3107-3112.

[31] E. Cerda, L. Mahadevan, Geometry and physics of wrinkling, Phys. Rev. Lett. 90 (7) (2003) 074302.

[32] K.V. Zakharchenko, M.I. Katsnelson, A. Fasolino, Finite temperature lattice properties of graphene beyond the quasiharmonic approximation, Phys. Rev. Lett. 102 (4) (2009) 046808.

[33] F. Scarpa, S. Adhikari, A.S. Phani, Effective elastic mechanical properties of single layer graphene sheets, Nanotechnology 20 (6) (2009) 065709.

[34] A. Politano, G. Chiarello, Probing the Young's modulus and Poisson's ratio in graphene/metal interfaces and graphite: a comparative study, Nano Res. 8 (2015) 1847-1856.

[35] G. Kresse, J. Furthmüller, Efficient iterative schemes for ab initio total-energy calculations using a plane-wave basis set, Phys. Rev. B 54 (16) (1996) $11169-11186$.

[36] P. Khomyakov, G. Giovannetti, P. Rusu, G. Brocks, J. Van den Brink, P. Kelly, First principles study of the interaction and charge transfer between graphene and metals, Phys. Rev. B 79 (19) (2009) 195425.

[37] J.P. Perdew, A. Zunger, Self-interaction correction to density-functional approximations for many-electron systems, Phys. Rev. B 23 (10) (1981) 5048-5079.

[38] J.P. Perdew, K. Burke, M. Ernzerhof, Generalized gradient approximation made simple, Phys. Rev. Lett. 77 (18) (1996) 3865-3868.

[39] S. Grimme, Semiempirical GGA-type density functional constructed with a long-range dispersion correction, J. Comput. Chem. 27 (15) (2006) 1787-1799.

[40] J. Klimeš, D.R. Bowler, A. Michaelides, Van der Waals density functionals applied to solids, Phys. Rev. B 83 (19) (2011) 195131.

[41] M. Dion, H. Rydberg, E. Schröder, D.C. Langreth, B.I. Lundqvist, Van der Waals density functional for general geometries, Phys. Rev. Lett. 92 (24) (2004) 246401.

[42] G. Román-Pérez, J.M. Soler, Efficient implementation of a van der Waals density functional: application to double-wall carbon nanotubes, Phys. Rev. Lett. 103 (9) (2009) 096102.

[43] T. Bucko, J. Hafner, S. Lebegue, J.G. Angyán, Improved description of the structure of molecular and layered crystals: ab initio DFT calculations with van der Waals corrections, J. Phys. Chem. A 114 (43) (2010) 11814-11824.

[44] Q. Lu, M. Arroyo, R. Huang, Elastic bending modulus of monolayer graphene, J. Phys. D Appl. Phys. 42 (10) (2009) 102002. 